\title{
N90-18991
}

\section{SEARCH FOR RESONANCES IN POSITRON-ATOM SYSTEMS}

\author{
A. K. Bhatia and Richard J. Drachman \\ Laboratory for Astronomy and Solar Physics \\ Goddard Space Flight Center \\ Greenbelt, MD 20771
}

\section{ABSTRACT}

No one likes to see a scattering cross-section curve that is too smooth; it is much more interesting to find bumps and wiggles and most interesting if it is possible to understand their cause. Several types of resonances have been clearly established in positroncontaining systems: those lying just below a degenerate threshold 1 (like $2 \mathrm{~s}$ $2 p$ in hydrogenic atoms or ions) and those representing Coulomb bound states in a re-arranged channe ${ }^{2}$ (like $\mathrm{Ps}+\mathrm{H}=$ $\left.\mathrm{e}^{+}+\mathrm{H}^{-}.\right)$Recently, two new sorts of resonances have been reported for which the resonant mechanism is not clear. The first ${ }^{3}$ is a very low-lying resonance in the e-Ps system (obtained by an adiabatic expansion method), and the second 4 is a similarly low-lying twochannel resonance in the $\mathrm{e}^{+-H}$ system (obtained by a close-coupling technique.) These developments encouraged us to examine such systems using the standard methods of stabilization and complex rotation. Most of our results are negative; 'we do not verify the low-lying resonances in either system. Some indication of new resonances in the $\mathrm{e}^{+}-\mathrm{He}^{+}$system is found; this may be caused by the attraction between $P s$ in the $n=2$ state and the $\mathrm{He}^{++}$nucleus.

METHOD

The Hamiltonian for the three-body systems of interest is the following

$$
-\sum_{i=1}^{3} \frac{1}{m_{i}} \nabla_{i}^{2}+\sum_{i<j=l}^{3} \frac{2 z_{i j}}{r_{i j}},
$$

where the three particles are indicated in an obvious way by subscripts. We then proceed to obtain approximate eigenvalues of this Hamiltonian by the usual variational technique, using a standard Hylleraas type of trial function.

If we were looking for bound states this would be the end of the story. We are, however, interested here in resonances; this changes the situation considerably. There are two ways to use the variational method in a search for resonances, and we use both. These are the stabilization and complex rotation methods. The first of these is the simpler one, and it is usual to apply it first; if an indication of possible resonant structure is found the second may then be applied.

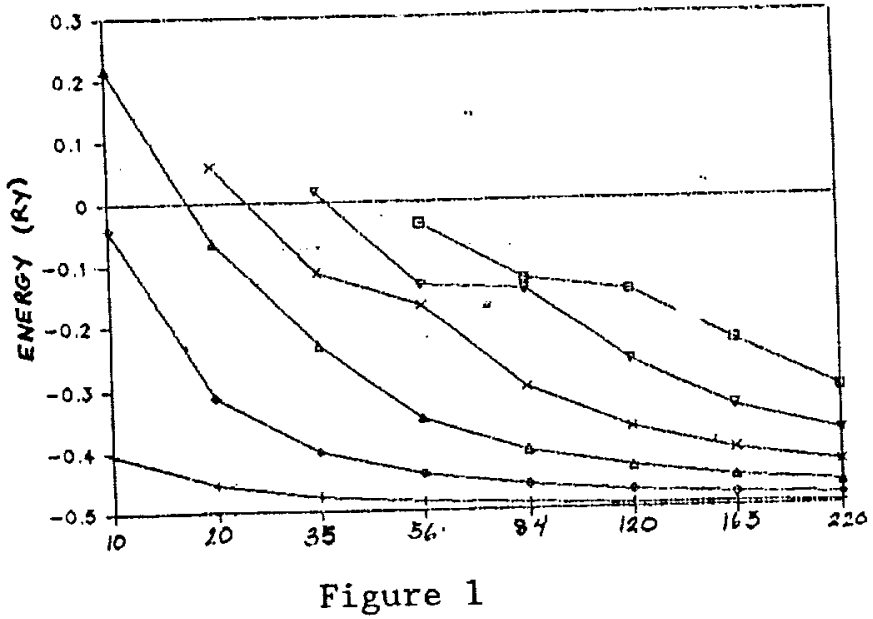

In the stabilization method one proceeds just as if one were looking for bound states but tries to find energy levels that are not very sensitive to the number of terms retained in the Hylleraas expansion. If there is a resonance at an energy where only a single channel is open, then a good indication would be an avoided crossing of two energy levels. A stabilized or 
slowly decreasing eigenvalue would be successively passed by rapidly decreasing energy levels. This is illustrated in Fig.1, which is the case of electron-positronium S-wave scattering in the electronic singlet state $\left(m_{i}=1, z_{12}=1, z_{13}=z_{23}=-1\right.$.) The clear avoided crossings just below the $n=2$ level of $P s$ at $E=-0.125$ Ry is the first of an infinite series of Feshbach resonances that are well understood to be due to the degeneracy of that level. In Ref.3 the existence of a resonance just above the elastic threshold is suggested; there is clearly no support for this in the present work.

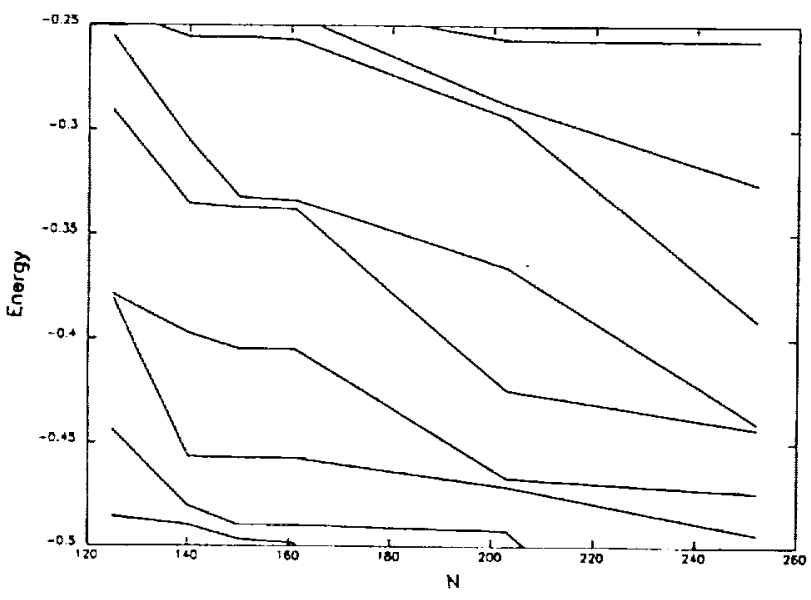

Figure 2

In Fig. 2 we show the same sort of diagram for the case of $\mathrm{S}$-wave positronhydrogen scattering $\left(m_{1}=m_{2}=1, m_{3}=\infty\right.$, $\mathrm{Z}_{12}=\mathrm{Z}_{23}=-1, \quad \mathrm{z}_{13}=1$.; Again it is clear that there is a stabilized eigenvalue just below the $n=2$ threshold in hydrogen corresponding to the first of an infinite series of Feshbach resonances 5 produced by the degeneracy of the $n=2$ levels. Although this resonance lies in a region where two channels are open $\left(\mathrm{e}^{+}-\mathrm{H}\right.$ and $\left.\mathrm{Ps}-\mathrm{H}^{+}\right)$there is no possibility of confusing the stabilized energy with an open threshold. Notice, however, that there is an indication of a second resonance just above the Ps threshold at $E=-0.5$ Rydberg. Is it possible that this corresponds to one of the resonances reported in Ref.4? Experience has taught us that it is most likely for an apparently stabilized energy lying above an open threshold to represent an ordinary elastic scattering state predominantly involving that particular channel; this is especially likely when there is no apparent mechanism for forming a resonance at that energy. (In this case only a shape resonance would be possible, and for $S$ wave scattering it is hard to see where an effective barrier in the potential could originate.) To be more certain of the situation we turn to the complex rotation method.

This method is based on carrying out the dilatation transformation

$$
r_{i} \rightarrow r_{i} e^{i \theta}
$$

which is equivalent to multiplying the potential energy part of Eq.1 by $e^{-i \theta}$ and the kinetic energy part by $e^{-2 i \theta}$. This analytically continued Hamiltonian is then diagonalized as before to obtain complex eigenenergies; since we use real basis functions the expansion coefficients must now be complex. If these energies are now plotted on the complex energy plane, they should behave as follows. True bound states are represented by points on the real axis, and ordinary scattering states are points that lie (in principle) along "rotated cuts" beginning at each target threshold on the real axis and making an angle $-2 \theta$ with that axis. Most importantly, points representing resonances, usually hidden on the second Riemann sheet, are revealed by the transformation; they should be independent of the angle $\theta$ and are complex. It is clear that true resonances should be well differentiated from ordinary scattering states, but in practice this requires quite large basis sets.

In Fig. 3 we show the energy plane for the $\mathrm{e}^{+}-\mathrm{H}$ system discussed above where $\theta=10.3^{\circ}$ and $N=161$. The cuts (rotated through an angle $-2 \theta$ ) 
corresponding to the first two thresholds in $\mathrm{H}$ and the ground state of Ps have been plotted, and one can see the points that approximately lie along them; the higher cuts are better represented in this case because of our particular choice of non-linear parameters in the trial function. There is no clear sign of an isolated point above the Ps threshold that might be a resonance although Doolen's resonance 5 is visible just below the $n=2$ threshold. Probably the "stabilized" energy that appeared in Fig.2 is in reality one of the points lying on the Ps cut; such points slide down the cut as $N$ is increased but slow down as they approach the threshold. This is the reason for doubting the reality of apparent resonances lying close above a target threshold.

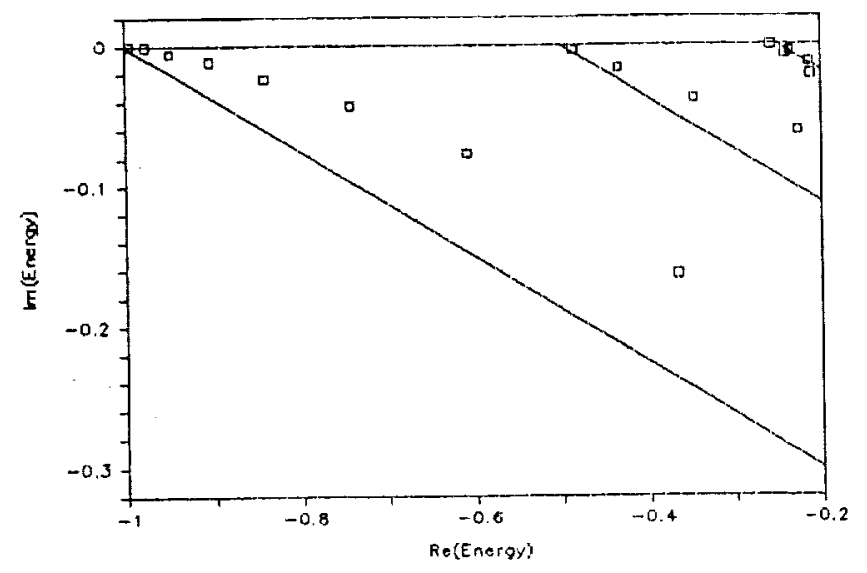

Figure 3

We made one final attempt to find simple new resonances by examining the $\mathrm{e}^{+}-\mathrm{He}^{+}$system. Although the same degeneracies exist as in the $\mathrm{e}^{+}-\mathrm{H}$ case, the repulsive Coulomb force is so dominant for large separations that the Feshbach resonances below target atom thresholds are unlikely to appear. But such resonances are probable below the degenerate thresholds in the $\mathrm{Ps}-\mathrm{He}^{++}$ channe1; because of the increased charge these should be lower lying than in the $\mathrm{Ps}-\mathrm{H}^{+}$case. So far, we have found indications of two such resonances at $E=-.73$ and $E=-.39$ Ry. These lie very far below their apparent "parent" threshold at $E=-.125$ Ry; the first is below the Ps ground state but above the two lowest states of $\mathrm{He}^{+}$. Our next attack on this problem will involve the Feshbach projection operator technique 6 which should be definitive.

1.Y. K. Ho, Phys. Lett. 120A, 348 (1984).

2.R. J. Drachman and S. K. Houston, Phys. Rev. A 12, 885 (1975); Y. K. Ho, Phys. Rev. A 17, 1675 (1978).

3.V. S. Melezhik and F. R. Vukajlovic, Phys. Rev. A 38, 6426 (1988).

4.M. Basu, M. Mukherjee, and A. S. Ghosh, J. Phys. B 22, 2195 (1989).

5.G. D. Doolen, J. Nuttall, and C. Wherry, Phys. Rev. Lett. 40, 313 (1978); L. T. Choo, M. C. Crocker, and J. Nuttal1, J. Phys. B 11, 1313 (1978).

6.H. Feshbach, Ann. Phys. (N.Y.) 19, 287 (1962) 
